\title{
COMPRESSIVE SENSING FOR HIGH RESOLUTION DIFFERENTIAL SAR TOMOGRAPHY - THE SL1MMER ALGORITHM
}

\author{
Xiao Xiang Zhu ${ }^{1}$, Richard Bamler ${ }^{1,2}$ \\ ${ }^{1}$ Technische Universität München, Lehrstuhl für Methodik der Fernerkundung, Arcisstraße 21, 80333, München, \\ Germany. Contact: xiao.zhu@dlr.de \\ ${ }^{2}$ German Aerospace Center (DLR), Remote Sensing Technology Institute (IMF), Germany
}

\begin{abstract}
Differential SAR tomography extends the synthetic aperture principle into the elevation and time directions for 4-D imaging. With modern meter-resolution space-borne SAR systems like TerraSAR-X (TS-X), systematic tomographic imaging of urban infrastructure and its deformations becomes feasible. We demonstrate the potential of TS-X data for this purpose and introduce several novel concepts.

Since building deformation in general is nonlinear, e.g. due to thermal dilation, we start from a tomographic system formulation that is general enough to allow for the inclusion of motion models (linear, periodic, etc.). By appropriate warping of the time axis we map the motion model function to become linear and lead to a peak in the spectral domain.

For the differential tomographic inversion itself we propose a 2-D compressive sensing (CS) based approach"SL1MMER". We demonstrate the super-resolution power and the robustness of SL1MMER both with simulated and with real data. We also show that it provides an attractive compromise between parametric and non-parametric methods. A full reconstruction of a building complex and its seasonal deformation from a stack of TS-X spotlight data is finally presented.
\end{abstract}

Index Terms-differential SAR tomography, nonlinear motion, compressive sensing, SL1MMER, super-resolution, TerraSAR-X

\section{INTRODUCTION}

Differential SAR Tomography (D-TomoSAR), also referred to as 4-D focusing, uses stacks of repeat-pass acquisitions to reconstruct reflectivity and deformation profiles of the scattering objects along elevation $s$ by means of 2-D spectral analysis for every azimuth-range $(x-r)$ pixel.

Currently, very high spatial resolution (VHR) SAR satellites like TerraSAR-X (TS-X) and COSMO-Skymed provide data up to $1 \mathrm{~m}$ resolution, which are particularly suited for tomographic imaging of urban infrastructure and their temporal deformations. Due to the repeat-pass nature of data acquisition, deformation terms must always be accounted for by the reconstruction algorithms. Most often pronounced seasonal thermal dilation prohibits the use of the popular linear motion assumption. In this paper we propose a model-based time warp method for nonlinear motion monitoring.

Due to the tight orbit control of these satellites the tomographic elevation resolution can be about 10-50 times worse than the one in azimuth or range. This extreme anisotropy calls for super-resolution algorithms. We work with TS-X spotlight data and concentrate on single-look super-resolution methods to exploit the potential of VHR data. All methods that require multi-look estimates of covariance matrices (e.g. CAPON or MUSIC) would reduce the azimuth-range resolution and are not able to resolve structural building elements in the important meter scale. In this paper a compressive sensing (CS) [1] based algorithm named "Scale-down by L1 norm Minimization, Model selection, and Estimation Reconstruction" (SL1MMER, pronounced "slimmer") for D-TomoSAR is proposed and we demonstrate its favourable properties like superresolution, robustness against phase noise, etc.

\section{SYSTEM MODEL}

The focused complex-valued measurement $g_{n}$ at an azimuth-range pixel for the $\mathrm{n}^{\text {th }}$ acquisition at aperture position $b_{n}$ and time $t_{n}(n=1, \ldots, N)$ is [2]:

$$
g_{n}=\int_{\Delta s} \gamma(s) \exp \left(-j 2 \pi\left(\xi_{n} s+2 d\left(s, t_{n}\right) / \lambda\right)\right) d s
$$

where $\gamma(s)$ represents the reflectivity function along elevation $s$ with an extent of $\Delta s$ and $\xi_{n}=-2 b_{n} /(\lambda r)$ is the spatial (elevation) frequency. $d\left(s, t_{n}\right)$ is the line-ofsight (LOS) deformation as a function of elevation and time. By introducing the temporal frequency $\eta_{n}=2 \tau_{n} / \lambda$ as a function of an artificial temporal baseline $\tau_{n}$ and a motion parameter $p(s)$, the proposed time warp method leads to a generalized system model which can be adapted for different nonlinear motion models:

$$
g_{n}=\int_{\Delta s} \gamma(s) \exp \left(-j 2 \pi\left(\xi_{n} s+\eta_{n} p(s)\right)\right) d s
$$

For instance, in case of linear motion, $\tau_{n}=t_{n}$ and the motion parameter $p(s)$ stands for the LOS velocity. In case of seasonal motion caused by thermal expansion, 
$\tau_{n}=\sin \left(2 \pi\left(t_{n}-t_{0}\right)\right)$ and the deformation parameter $p(s)$ stands for the amplitude of the periodic motion along $s ; t_{0}$ is the initial phase offset which can be estimated from the temperature history. After the time warp, the system model (2) can be easily rewritten as:

$$
g_{n}=\int_{\Delta s} \int_{\Delta p} a_{\gamma}(s, p) \exp \left(-j 2 \pi\left(\xi_{n} s+\eta_{n} p\right)\right) d s d p
$$

where $a_{\gamma}(s, p)=\gamma(s) \delta(p-p(s)) \quad$ is the scattering distribution in the elevation-motion $(s-p)$ plane. Equation (3) is a 2-D Fourier transform of $a_{\gamma}(s, p)$ which is a deltaline in the elevation-motion $(s-p)$ plane along $p=p(s)$. Its projection onto the elevation axis is the reflectivity profile $\gamma(s)$. This model is a generalization of the one introduced in [2]. For the following we use a discretized version of equation (3), i.e. a 2-D discrete Fourier transform:

$$
\mathbf{g}=\mathbf{R} \gamma
$$

where $\mathbf{g}$ is the measurement vector with $N$ elements, $\mathbf{R}$

with $\quad R_{n \times(l+L q)}=\exp \left(-j 2 \pi\left(\xi_{n} s_{l}+\eta_{n} p_{q}\right)\right) \quad$ is $\quad$ an $\quad \mathrm{N} \times \mathrm{LQ}$ mapping matrix, $\gamma$ is the discrete $a_{\gamma}(s, p)$ in elevation $s_{l}(l=1, \ldots, L)$ and motion $p_{q}(q=1, \ldots, Q)$.

\section{THE SL1MMER ALGORITHM}

Sparse tomographic SAR inversion requires the estimation of the number of scatterers, as well as the amplitude, phase, elevation, and motion parameter of each scatterer. As will be shown, the SL1MMER algorithm estimates these parameters in a very accurate and robust way. It consists of three main steps: 1) a dimensionality scale-down by L1 norm minimization, 2) model selection and 3) parameter estimation.

\section{- Scale-down by $\mathbf{L}_{\mathbf{1}}$ norm minimization}

Since $L Q \gg N$, the system model (4) is severely underdetermined. Hence, there are infinitely many solutions. As described in the introduction and outlined in detail in [3], $\gamma$ is sparse in the object domain for VHR space-borne X-band TomoSAR with typically 1-4 point-like contributions of unknown positions, amplitudes and phases, i.e. $\gamma$ contains only 1-4 non-zero elements. This sparsity property of $\gamma$ suggests using the compressive sensing approach. It says, if the mapping matrix fulfills certain properties, i.e. the restricted isometry property (RIP) and the incoherence property [1], with number of measurements depending on the sparsity $\mathrm{K}$ instead of the length of the signal LQ, the sparse signal $\gamma$ can be very well approximated by the solution with the least number of scatterers (non-zero elements of $\gamma$ ), i.e. the minimal $\mathrm{L}_{0}$ norm, which satisfies the measurements with noise. In addition, if $N \geq \mathrm{O}(K \log (L / K))$, the convex $\mathrm{L}_{1}$ norm minimization gives the same solution as the N-P hard $\mathrm{L}_{0}$ norm minimization. In case there is no prior knowledge about $\mathrm{K}$ and in the presence of measurement noise, it can be approximated by a global optimization of an object function consisting of an $\mathrm{L}_{2}$ norm residual term and an $\mathrm{L}_{1}$ norm regularization term. For VHR space-borne X-band TomoSAR, where $\mathrm{K} \leq 4$, the reconstruction of $\gamma$ boils down to the following $\mathrm{L}_{1}-\mathrm{L}_{2}$ norm minimization:

$$
\hat{\gamma}=\arg \min _{\gamma}\left\{\|\mathbf{h}-\mathbf{R} \gamma\|_{2}^{2}+\lambda_{K}\|\gamma\|_{1}\right\}
$$

where $\lambda_{K}$ is the Lagrange multiplier as a function of $\mathrm{N}$ and $\varepsilon$. The minimization of (4) gives a robust estimate of the plausible positions of the scatterers, among which there might be a few outliers contributed by noise. By only selecting its columns corresponding to the non-zero elements of $\hat{\gamma}$, the mapping matrix $\mathbf{R}$ is scaled down significantly. It renders the severely under-determined system model finally over-determined.

\section{- Model selection}

The $\mathrm{L}_{1}-\mathrm{L}_{2}$ norm minimization step shrinks $\mathbf{R}$ dramatically and gives a first sparse estimate of $\gamma$. This estimate, though, may still contain outliers and, hence, the sparsity $K$ (i.e. the number of the scatterers) is often overestimated. Model selection is used to clean the $\gamma$ estimate of spurious, nonsignificant scatterers and to finally obtain the most likely number $\hat{K}$ of scatterers inside a resolution cell. It provides a trade-off between the model complexity and the model fit. The implementation detail of different model selection schemes for VHR TomoSAR is addressed in detail in [5].

\section{- Parameter estimation}

As a last refinement, a much slimmer $N \times \hat{K}$ mapping matrix $\mathbf{R}(\hat{\mathbf{s}}, \hat{\mathbf{p}})$, with $\mathbf{R}_{n, k}(\hat{\mathbf{s}}, \hat{\mathbf{p}})=\exp \left(-j 2 \pi\left(\xi_{n} \hat{s}_{k}+\eta_{n} \hat{p}_{k}\right)\right)$, is built up, and the final complex-valued reflectivity for each scatterers $\hat{\gamma}(\hat{\mathbf{s}}, \hat{\mathbf{p}})$ is obtained by :

$$
\hat{\gamma}(\hat{\mathbf{s}}, \hat{\mathbf{p}})=\left(\mathbf{R}^{H}(\hat{\mathbf{s}}, \hat{\mathbf{p}}) \mathbf{R}(\hat{\mathbf{s}}, \hat{\mathbf{p}})\right)^{-1} \mathbf{R}^{H}(\hat{\mathbf{s}}, \hat{\mathbf{p}}) \mathbf{g}
$$

By introducing model selection and final refinement of the parameter estimation, SL1MMER improves the result of CS by means of removing possible outliers and providing more accurate amplitude and phase estimates.

\subsection{The Data Set}

\section{EXPERIMENTS}

We work with TS-X spotlight data with a resolution of $0.6 \mathrm{~m}$ in slant range and $1 \mathrm{~m}$ in azimuth. Our test site is Las Vegas, Nevada, USA. The orbit of TS-X is controlled in a tube of $500 \mathrm{~m}$ diameter. The data stack consists of 25 scenes with an elevation aperture size $\Delta b=269.5 \mathrm{~m}$. With non-parametric linear spectral analysis based on regularized SVD [2][5] we obtain a $3 \mathrm{~dB}$ elevation resolution of $\rho_{s}=33 \mathrm{~m}$ or about $16 \mathrm{~m}$ in height $z$ (look angle $=31.8^{\circ}$ ). The Cramér-Rao lower bound (CRLB) of the elevation estimates of a single scatterer is $1.1 \mathrm{~m}$ for a $\mathrm{SNR}=10 \mathrm{~dB}$ in this configuration [5]. 


\subsection{Simulation}

In this section, CS, as the spectral estimator of SL1MMER, is compared to conventional non-parametric and parametric methods using simulated data with the elevation sampling of the real data. Decorrelation is introduced by adding Gaussian noise with variable SNR. Phase noise due to unmodeled motion and atmospheric effects is simulated by adding a uniformly distributed phase.

Figure 1 shows the 4-D reconstruction with linear motion using a singular value decomposition method with Wienertype regularization (SVD-Wiener) [5] (left) and CS (right). We simulate the situation of two scatterers inside one resolution cell with elevation of $0 \mathrm{~m}$ and $20 \mathrm{~m}$ and linear deformation velocity of $0.9 \mathrm{~cm} / \mathrm{y}$ and $1.1 \mathrm{~cm} / \mathrm{y}$, respectively. SVD-Wiener is not able to distinguish them while CS detects very clearly two individual scatterers. Figure 2 shows the same plot as Figure 1 but with seasonal motion. The two scatterers are far apart from each other with elevation of -30 and $20 \mathrm{~m}$ and seasonal motion amplitude of $8 \mathrm{~mm}$ and $4 \mathrm{~mm}$. With time warp, the amplitudes of seasonal motion can be well reconstructed.
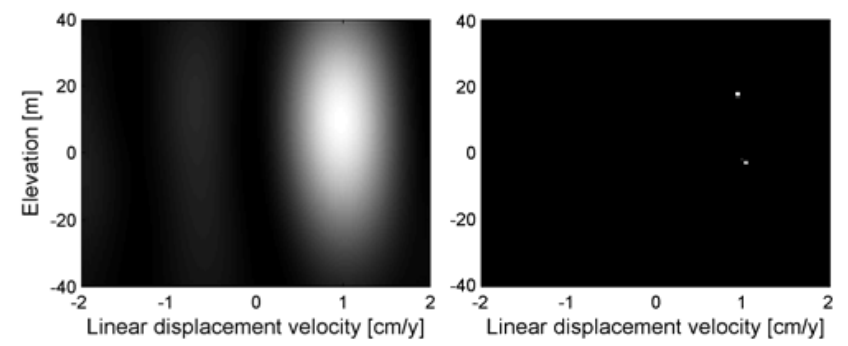

Figure 1: 4-D reconstruction with linear motion: SVD-Wiener (left) vs. $\mathrm{CS}$ (right). $\mathrm{SNR}=10 \mathrm{~dB} ; \mathrm{s}=0,20 \mathrm{~m} ; \mathrm{v}=0.9 \mathrm{~cm} / \mathrm{y}, 1.1 \mathrm{~cm} / \mathrm{y}$.
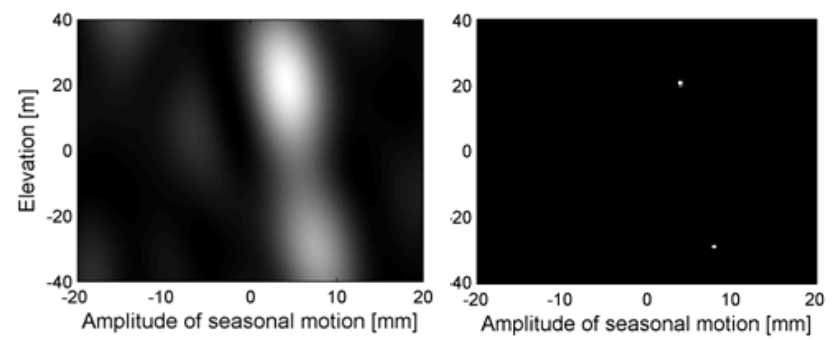

Figure 2: 4-D reconstruction with seasonal motion: SVD-Wiener (left) vs. CS (right). SNR $=10 \mathrm{~dB} ; \mathrm{s}=-30 \mathrm{~m}, 20 \mathrm{~m}$; amplitude of seasonal motion $=8 \mathrm{~mm}, 4 \mathrm{~mm}$.

In [3], CS has been compared to maxima detection (MD) and NLS, where MD simply uses the maxima of the SVDWiener reconstruction as estimates and NLS is the theoretically best solution under Gaussian noise. Compared to $\mathrm{MD}$, besides the super-resolution property, CS shows no sidelobe interference. Compared to NLS, CS has comparable performance with lower computational effort and does not require the number of scatterers as a prior. The left plot of Figure 3 shows the elevation estimation accuracy of a single scatterer in the phase-noise-free case using NLS and CS compared to the CRLB as a function of SNR. The estimation accuracy of CS is almost identical to NLS and reaches the CRLB. The right plot of Figure 3 shows the elevation error under phase noise uniformly distributed in $\left[-\phi_{n}, \phi_{n}\right)$. Obviously CS is more robust against nonGaussian phase noise than NLS. Taking all those aspects into account, CS provides the best of the two worlds of nonparametric and parametric spectral estimation methods and, hence, is proven very attractive for D-TomoSAR.
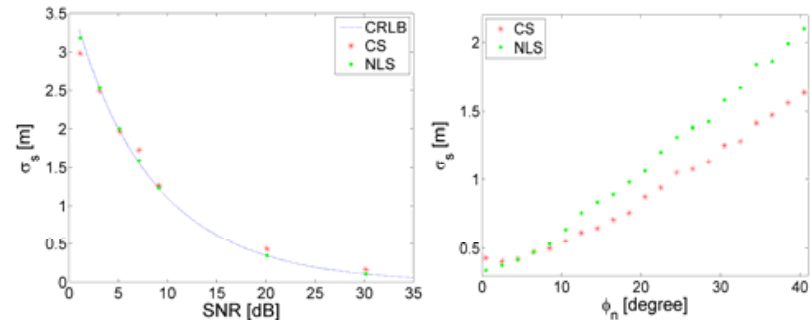

Figure 3: left: Single scatterer elevation estimation error of NLS and CS compared to the CRLB as a function of SNR. Right: Single scatterer elevation estimation error of NLS and CS as a function of phase noise $(\mathrm{SNR}=20 \mathrm{~dB})$.

\subsection{Real Data}

We use the Las Vegas convention center as a test site. It has a height of about $20 \mathrm{~m}$, roughly the resolution limit of SVDWiener for our elevation aperture size. The left image in Figure 4 shows the TS-X intensity map. The presence of two scatterers within an azimuth-range pixel is expected in layover areas and has been validated in [5]. Thus, we are able to compare the performance of CS to SVD-Wiener in the layover areas. Figure 5 shows the projections of the 4-D reconstruction for the pixel $\mathrm{P}$ (red dot) to elevation direction, i.e. the reflectivity profile. Two scatterers with slightly different velocities have been detected by SVDWiener (red line), one on the roof, the other on the parking place on the ground. The blue line in Figure 4 shows the result using CS. Two very close scatterers have been detected, i.e. D-TomoSAR via CS provides super resolution up to $2 \mathrm{~m}$ in height (i.e. about $4 \mathrm{~m}$ in elevation) in this case.

With the approximately one year time spread of our data set, nonlinear (e.g. thermally induced) movements of different building parts must be expected. Hence, by using our time warp method, the surface model and amplitude map of seasonal motion is obtained for the whole building. The center image of Figure 4 shows the surface model generated from the elevation estimates (converted to height). The full structure of the convention center has been captured at a very detailed level. Besides the building, more detail such as the roads surrounding the convention center, as well as two bridges above the roads which have weak but correlated returns are clearly resolved. The height estimates are very precise compared to the $33 \mathrm{~m}$ elevation resolution due to the high SNR of TS-X data. The right image of Figure 4 represents the amplitude map of the seasonal motion. The amplitude variance is smooth for individual structural blocks with sudden amplitude changes between adjacent blocks. The amplitude difference is up to $8 \mathrm{~mm}$. 

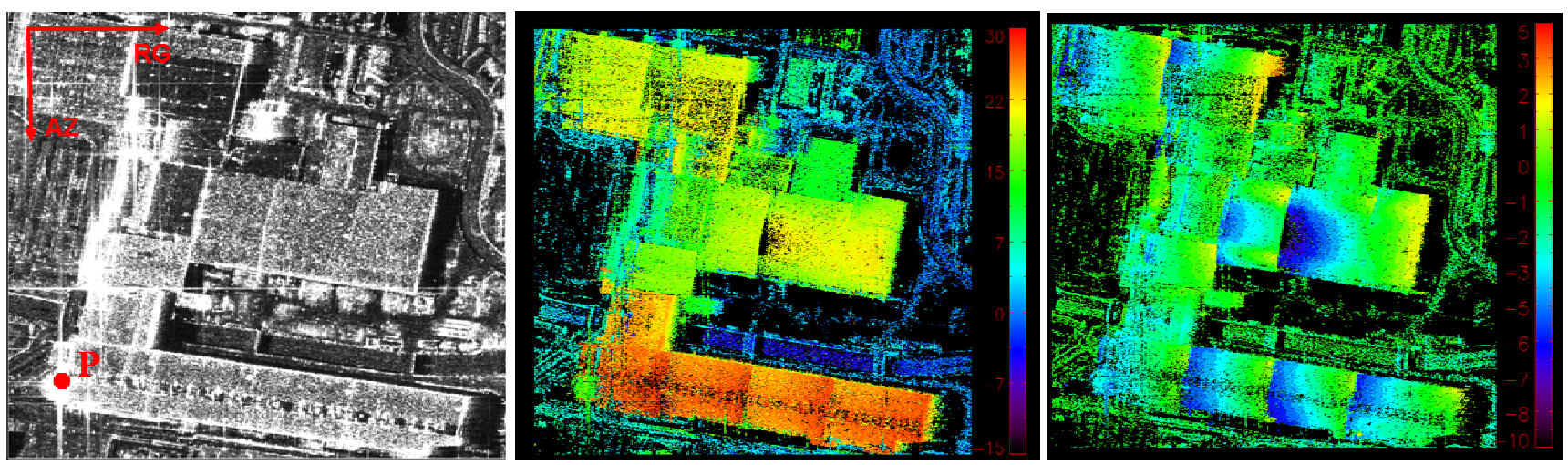

Figure 4: Left: TS-X intensity map of Las Vegas convention center; middle: Reconstructed digital surface model (DSM) from D-TomoSAR, [unit: $\mathrm{m}$ ]; right: Estimated amplitude of seasonal motion using the time warp method [unit: $\mathrm{mm}$ ]

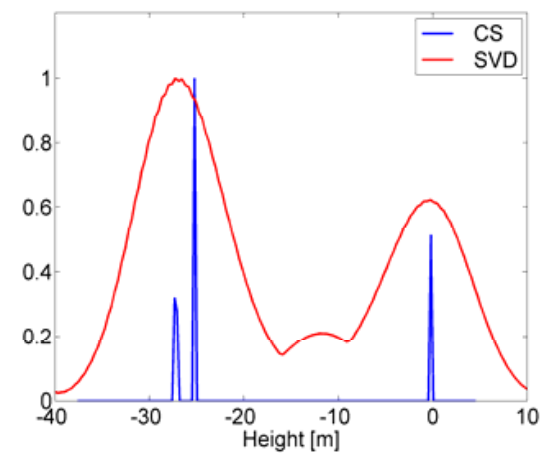

Figure 5: Reflectivity reconstruction for a pixel located at the layover area by using SVD-Wiener (red) and CS (blue).

\section{CONCLUSION AND OUTLOOK}

The new class of space-borne high resolution spotlight SAR data is very attractive for 3-D and 4-D tomographic mapping of urban infrastructure. Compared to the medium resolution SAR systems available so far, the information content and level of detail has increased dramatically.

Recognizing the sparsity of the signal in elevation, as a new and promising technique for sparse signal reconstruction, CS based SL1MMER algorithm has been proposed to DTomoSAR. It provides a very elegant compromise between conventional non-parametric and parametric tomographic methods. For instance, it shows high robustness w.r.t. unmodeled non-Gaussian phase noise. Compared to nonparametric methods, it provides super-resolution properties without sacrificing the azimuth or range resolution; meanwhile, it does not suffer from the sidelobe interference effect. Compared to parametric methods, like NLS, in the single scatterer case and under Gaussian noise SL1MMER approaches the accuracy of NLS with lower computational effort. In addition, it does not require the number of scatterers inside a resolution cell as a prior.

A new model-based time warp method has been proposed for nonlinear motion monitoring. By forming an artificial temporal baseline, it provides the possibility of focusing the desired parameter, e.g. the amplitude of seasonal motion, to the coefficient space. The time warp method has been validated by reconstructing seasonal motion caused by thermal expansion of a building complex. A full tomographic high resolution reconstruction of the Las Vegas convention center is presented.

Further work will focus on evaluating the super-resolution power and robustness of the SL1MMER algorithm [6], i.e. trying to find its limits.

\section{REFERENCES}

[1] E. Candès, Compressive sampling, Int. Congress of Mathematics, 3, pp. 1433-1452, Madrid, 2006

[2] G. Fornaro, D. Reale, F. Serafino, Four-Dimensional SAR Imaging for Height Estimation and Monitoring of Single and Double Scatterers, IEEE Trans. Geosci. Remote Sensing, Vol. 47, pp: 224 - 237, 2009

[3] X. Zhu, R. Bamler, Tomographic SAR Inversion by L1 Norm Regularization - The Compressive Sensing Approach, IEEE Transactions on Geoscience and Remote Sensing, accepted.

[4] S. Chen, D. Donoho, M. Saunders, Atomic Decomposition by Basis Pursuit, SIAM Journal on Scientific Computing, Vol. 20, pp.33-61,1998

[5] X. Zhu, R. Bamler, Very High Resolution Spaceborne SAR Tomography in Urban Environment, IEEE Transactions on Geoscience and Remote Sensing, accepted.

[6] X. Zhu, R. Bamler, Super-Resolution Power and Robustness of Compressive Sensing for Spectral Estimation with Application to Spaceborne Tomographic, IEEE Transactions on Geoscience and Remote Sensing, submitted. 